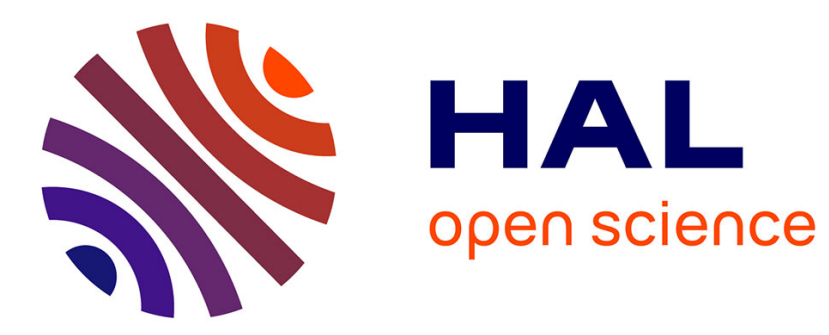

\title{
Les apprentissages des élèves dans leur contexte: les effets de la composition de l'environnement scolaire
}

Marie Duru-Bellat

\section{To cite this version:}

Marie Duru-Bellat. Les apprentissages des élèves dans leur contexte: les effets de la composition de l'environnement scolaire. Carrefours de l'éducation, 2003, 16, pp.183-206. halshs-00004912

\section{HAL Id: halshs-00004912 \\ https://shs.hal.science/halshs-00004912}

Submitted on 10 Oct 2005

HAL is a multi-disciplinary open access archive for the deposit and dissemination of scientific research documents, whether they are published or not. The documents may come from teaching and research institutions in France or abroad, or from public or private research centers.
L'archive ouverte pluridisciplinaire $\mathbf{H A L}$, est destinée au dépôt et à la diffusion de documents scientifiques de niveau recherche, publiés ou non, émanant des établissements d'enseignement et de recherche français ou étrangers, des laboratoires publics ou privés. 


\title{
Les apprentissages des élèves dans leur contexte : les effets de la composition de l'environnement scolaire
}

\author{
$\Delta$ Marie Duru-Bellat \\ université de Bourgogne et Institut de recherche sur l'éducation (IREDU-CNRS).
}

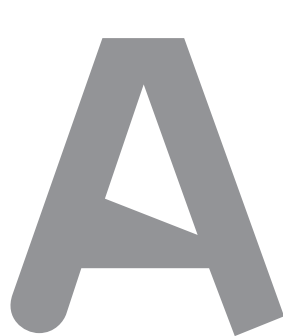

lors qu'on accorde à reconnaître le caractère contextualisé des apprentissages des élèves, l'influence de paramètres aussi essentiels du contexte que sa composition scolaire ou sociale reste encore assez peu explorée. Certes, la mouvance du school effectiveness démontre amplement l'influence de l'école ou de la classe fréquentées sur les progressions des élèves. Mais dans sa recherche de facteurs généraux d'efficacité, ce courant s'est peu interrogé sur leurs relations avec les caractéristiques du public d'élèves. Pourtant tous les travaux sur l'impact des modes de groupement des élèves montrent que tant le niveau moyen du groupe que sa composition sociale (ce que les AngloSaxons désignent sous le vocable de school mix) affectent les progressions moyennes et également les inégalités entre élèves. Cette influence passe par les modulations que suscite le mode de groupement d'une part dans la quantité et la qualité de l'instruction délivrée en classe, d'autre part quant aux attitudes et aux comportements des élèves affectés aux différents groupes. 


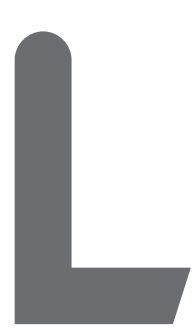

es chercheurs en éducation mettent volontiers en avant, c'est même ainsi, parfois, qu'ils se distinguent des psychologues, le caractère contextualisé des apprentissages des élèves, contextualisé dans une classe, dans un établissement. Ils rejoignent ainsi un postulat classique de la sociologie, à savoir que l'environnement social influe sur les conduites individuelles, au-delà des caractéristiques personnelles des acteurs. Depuis ses débuts, la sociologie de l'éducation s'est interrogée sur l'existence d'effets contextuels, c'est-à-dire tenant spécifiquement aux caractéristiques de l'environnement social (environnement scolaire, quartier et communauté d'appartenance...) sur les aspirations et la réussite des élèves. C'est ainsi par exemple que le sociologue américain Wilson (1959) prend explicitement en compte, dans ses analyses des projets de jeunes lycéens, l'appartenance sociale définie à la fois au niveau individuel et au niveau de l'environnement scolaire, en insistant sur la pression normative du groupe d'appartenance et l'impact de ses valeurs sur ses membres. On a bien affaire en l'occurrence à la recherche d'un effet spécifique du groupe, comme agrégation d'individus, dont il marque les conduites au-delà des effets tenant aux caractéristiques personnelles. Les travaux de Coleman (1966), dont l'influence sur l'agenda de la sociologie de l'éducation américaine sera très importante, abordent cette même question, mais en étant plus novateurs sur le plan de la méthode, pour dissocier facteurs individuels et facteurs contextuels, qu'en ce qui concerne l'élucidation des processus qui génèrent ces effets de contexte eux-mêmes. À partir des années soixante, vont coexister des travaux cherchant avant tout à évaluer les effets du contexte scolaire de manière externe en particulier la mouvance du school effectiveness, sur laquelle nous reviendrons, et des travaux plus qualitatifs tels que ceux de Campbell et Alexander (1965), affirmant que les analyses des effets structurels doivent «se déplacer, des caractéristiques du système dans sa globalité, aux situations sociales auxquelles les individus se trouvent confrontés ». Cette perspective invitera à explorer en particulier les voies par lesquelles la composition sociale d'un groupe, d'une classe ou d'un établissement, influe sur les comportements des élèves, avec un développement des travaux sur le school mix, que nous présenterons dans la dernière partie de ce texte.

Par rapport à cette riche tradition sociologique, le silence des grandes théories françaises sur l'école (tant Bourdieu et Passeron que Boudon) sur l'importance des facteurs de contexte dans l'explication des inégalités sociales de carrières scolaires est frappant. Certes, le contexte est bien perçu comme exerçant une influence, mais dans sa dimension macrosociale, au niveau des structures, sans que soient pris en compte les contextes plus proches et plus concrets, dont l'exploration ne s'esquissera qu'à partir des années quatre-vingt. Aujourd'hui, cette question de recherche n'a pas perdu de son actualité scientifique, comme en attestent les nom- 
breux travaux parus dans la dernière décennie, mais toujours plus abondants dans les pays anglo-saxons que dans la sociologie française.

Cette question de recherche revêt par ailleurs une actualité politique, comme le révèlent de manière récurrente les débats sur l'école. Ainsi, le succès du thème de l'hétérogénéité des élèves révèle la conviction que le contexte scolaire proche fait des différences: en l'occurrence l'hétérogénéité du public affecterait les progrès des élèves, plus précisément serait préjudiciable aux meilleurs et enfoncerait encore plus les faibles. Dans le même temps pourtant, la ségrégation, sociale et ethnique, qui caractériserait de plus en plus les établissements scolaires (évolution au demeurant ténue, cf. Trancart, 1998), est, elle aussi, dénoncée. Cette dénonciation ne se fait pas seulement en référence avec l'idéal de l'école républicaine intégratrice, mais aussi parce que l'homogénéité des contextes qu'elle entraîne creuserait les écarts, de progression et de qualité de l'«expérience scolaire» entre les élèves. On peut aussi citer le thème du choix de l'école, pourfendu par les uns mais défendu secrètement (en France) par beaucoup, qui s'appuie sur la conviction selon laquelle l'établissement fréquenté joue sur le devenir ou le vécu scolaire de l'élève. La question de l'importance du contexte sur le devenir scolaire est donc bien sous-jacente à nombre de préoccupations de politique éducative, ce qui justifie que l'examine avec soin l'éclairage de la recherche.

Ce texte présentera dans une première partie les travaux appréhendant les «effets établissements » et les débats qu'ils ont suscités. Puis, nous nous focaliserons sur les effets de la composition scolaire et sociale du public d'élèves, en abordant successivement son impact aux niveaux de l'établissement, de la classe et du groupe.

\section{$L$ a recherche sur les "effets établissements": acquis, débats et zones d'ombre}

Il s'agit en l'occurrence d'évaluer 1) dans quelle mesure les phénomènes scolaires (réussite, socialisation et orientation scolaires) subissent l'influence des caractéristiques du contexte environnant et quel est le «niveau de contexte » pertinent à cet égard (classe ou établissement notamment), 2) les processus par lesquels cette structure sociale exerce son effet sur les comportements et les attitudes individuelles.

Les contextes classe ou établissement sont évidemment différents sous de multiples dimensions, et la question est de savoir quelles sont les dimensions pertinentes. Dans les pays les plus pauvres par exemple, les contraintes économiques, qui déterminent les conditions matérielles d'enseignement, peuvent s'avérer dirimantes, alors qu'au contraire, elles seraient «suffisamment bonnes» (et uniformes) dans les pays riches pour ne plus exercer d'influence significative (au-delà donc du seuil où elles se situent). Il faut donc rappeler à ce propos que l'évaluation des effets de tel ou tel aspect du contexte, comme l'évaluation en général, est nécessairement comparative: on ne peut évaluer l'impact d'un facteur uniformément 
répandu, et par conséquent, on n'évalue de fait que ce qui varie. Ainsi, l'influence éventuelle de la composition sociale du public scolaire n'apparaîtra que dans les pays où elle varie d'un site à l'autre. Dans la même perspective, il faut souligner que la notion même d'école efficace est nécessairement relative, l'apport de la recherche étant de démontrer que, au sein d'un échantillon donné d'écoles, certaines écoles s'avèrent plus efficaces que d'autres.

L'investigation empirique de l'influence du contexte scolaire sur les résultats ou les attitudes des élèves remonte aux explorations pionnières de Coleman à la fin des années soixante concluant à l'absence d' « effets école» dans le contexte américain - le fameux schools make no difference -. Mais Coleman soulignait aussi que la seule caractéristique des écoles semblant exercer un effet significatif sur les progressions des élèves était leur composition raciale, les enfants des minorités ethniques réussissant mieux dans les écoles déségréguées; il mettait aussi en avant le fait que les différentes facettes des effets du contexte (sur les progressions ou sur la socialisation) pouvaient diverger: ainsi, dans ces contextes déségrégués où ils réussissaient plutôt mieux, les enfants des minorités ethniques avaient dans le même temps une image d'eux-mêmes plutôt moins bonne. Un temps, la prégnance des thèses centrées sur la notion de «handicap socioculturel» aidant, c'est le school make no difference qui a été surtout retenu, avec à la clef, de la part de l'école, une certaine résignation à l'impuissance.

Depuis les années quatre-vingt, par un mouvement de balancier qu'il est hors de propos d'analyser ici (cf. Thrupp, 1995), le courant de recherche dit de la school effectiveness a contribué à imposer l'idée d' « effets établissements » significatifs, minimisant du même coup les effets individuels et agrégés (dans un contexte) des caractéristiques individuelles (notamment sociales) des élèves. Dans cette perspective, quelles que soient les caractéristiques du public d'élèves, toute école peut améliorer son efficacité et son équité. Il s'agit donc clairement d'une perspective tournée vers l'action, en l'occurrence vers le school improvement. On entend réagir contre la démoralisation des enseignants, que nourrit le déterminisme des travaux sociologiques soulignant le poids de l'origine sociale des élèves, tout en rencontrant de fait une mouvance politique conservatrice (ou du moins critique par rapport à l'efficacité des institutions publiques) qui enjoint aux écoles de rendre des comptes sur leurs résultats, la question de l'inégalité des chances cédant alors le pas devant celle de l'efficacité des pratiques pédagogiques.

Le courant de recherche de la school effectiveness a développé cette problématique à partir des années quatre-vingt, de manière systématique, souvent sur la base de grandes enquêtes. Il s'agit d'un courant majoritairement anglo-saxon. En particulier, la recherche française est sur ces questions globalement très peu diserte. Plusieurs raisons expliquent ce «retard » et elles ont été analysées par ailleurs (Cousin, 1993). Soulignons simplement que se sont conjugués en France d'une part un contexte théorique fort, la théorie de la reproduction amenant à juger naif d'escompter qu'une action pédagogique locale puisse enrayer le mécanisme puissant 
de la reproduction sociale, et d'autre part des méthodologies courantes bien trop frustres pour appréhender la notion même d'effet de contexte (effet établissement et effet classe), qui ne peut être pensée et a fortiori évaluée sans une instrumentation de type analyse multivariée.

\section{Ce qui fait l'efficacité d'une école}

Dans les pays anglo-saxons où ont pris place de nombreux travaux empiriques, un certain nombre de "régularités» stables ont été produites ( $c f$. la synthèse de Scheerens, 2000). L'existence d' «effets établissement» significatifs est, dans ces pays, aujourd'hui démontrée, en matière de progression académique (les effets sur les attitudes étant moins explorés). Ces effets sont d'autant plus forts que l'école a le monopole des apprentissages en la matière, et se trouve donc moins concurrencée par d'autres instances comme la famille (les matières scientifiques dans le premier cas, la langue maternelle dans le second). Ces «effets établissement » sont d'importance modérée ${ }^{1}$ : selon les recherches, entre 8 et $15 \%$ de la variance des scores des élèves s'explique par les différences entre écoles (Elliott, 1996), la plupart des estimations se situant néanmoins dans le bas de cette fourchette (sur la base de méta-analyses anglaises et néerlandaises, Wyatt, 1996, conclut ainsi à un chiffre de 8 à $9 \%$ ). Ces effets école sur les progressions des élèves sont sans doute moins forts en primaire que dans le secondaire, mais il faut souligner que l'on n'évalue jamais que l'effet de ce qui varie d'un établissement à l'autre (le constat précédent s'expliquant alors, du moins en partie, par la plus grande variété des écoles secondaires). De même, si les «effets établissement» sont plus ténus en France que dans les pays voisins (proches de $5 \%$; cf. Grisay, 1997), c'est en partie du fait de leur plus grande homogénéité sur tout le territoire, comparativement à de nombreux pays industrialisés.

La recherche donne une idée assez précise de ce à quoi ressemble un collège ou une école efficaces; mais il est plus difficile de trancher sur ce qui fait qu'un établissement est plus performant qu'un autre, car la plupart de ces travaux sont de type corrélationnel: on sait seulement que telle caractéristique pédagogique est en moyenne associée à un «effet établissement» positif. Cinq facteurs s'avèrent en tout cas liés à de meilleurs résultats chez les élèves, sachant que ces résultats ont été établis majoritairement sur des écoles primaires, le plus souvent des écoles plutôt

1. L'ampleur des effets établissement est relative à la méthode statistique retenue; rappelons que les modèles les plus couramment utilisés, les modèles par les moindres carrés ordinaires, simplifient la réalité en faisant comme si l'effet établissement était le même quels que soient les groupes d'élèves considérés, alors que les caractéristiques du contexte peuvent jouer en interaction avec les caractéristiques individuelles. Ces modèles tendent à surestimer les effets des variables de contexte (cela ne vaut pas quand on raisonne en termes de pourcentage de variance expliquée). Si les modèles multiniveaux sont en théorie plus corrects dès lors que les données ont une structure hiérarchisée, ils introduisent d'autres hypothèses, sont d'un maniement plus complexe, et le sens des effets des différentes variables ne sont pas très différents (Jarousse et Leroy-Audoin, 1999). 
défavorisées des centres-villes. Ce sont une forte emprise du chef d'établissement, des attentes élevées à l'égard des élèves, une polarisation sur les acquis de base, un climat de sécurité et d'ordre, des évaluations fréquentes des progrès des élèves. À ces facteurs de base, s'ajoutent d'autres traits tels que la cohésion au sein de l'équipe éducative, la qualité des curricula (ou les chances d'apprendre qui sont données aux élèves), l'implication des parents, une gestion stricte du temps scolaire pour maximiser le temps de travail des élèves. Ces facteurs peuvent bien sûr se décliner de manière variable selon les établissements, mais ils se dégagent de manière stable des méta-analyses internationales (Scheerens, 2000). En France (Grisay, 1997), le rôle du chef d'établissement n'apparaît pas aussi nettement. Mais on retrouve, en premier lieu, une forte «exposition à l'apprentissage », avec une utilisation optimale du temps scolaire (peu de temps perdu pour la gestion de la discipline, notamment) et peu d'absentéisme. Jouent également des attentes élevées de la part des enseignants, et donc une valorisation marquée du travail scolaire, attentes élevées qui sont partagées avec les parents et les élèves. Interviennent aussi positivement la qualité des relations entre enseignants et élèves et de la vie au collège (telle qu'estimée tant par les élèves que par les enseignants), la clarté des règles, l'existence de droits et de responsabilités pour les élèves, un climat paisible. Ces divers facteurs (notamment une bonne ambiance, une bonne gestion du temps et le respect de la discipline) sont particulièrement importants pour les élèves en difficulté au début du collège (Trancart, 1993).

Dans la sociologie (qualitative) française, on insiste sur le fait que, de manière générale, c'est le «climat » de l'établissement qui s'avère important, notamment les dispositions générales des enseignants par rapport aux élèves, bien plus que sa politique déclarée, ou ses initiatives particulières, en matière d'innovation pédagogique en particulier.

Cette notion de climat reste à l'évidence difficile à cerner. Certes, dans la perspective d'une sociologie des organisations, des paramètres tels que les modalités de la division du travail, les dispositifs de régulation, le degré de cohésion interne (etc.) sont a priori importants ${ }^{2}$. Mais peu de travaux confrontent systématiquement ces paramètres avec ce que l'établissement «produit ». Néanmoins, il semble qu'au niveau collège la capacité des établissements à construire une politique d'ensemble, à se mobiliser autour d'un projet soit associée à une moindre sélectivité des cursus et une équité plus grande, notamment parce que les enseignants partagent une vision plus positive des élèves et de leur propre métier (Dubet et al., 1989; Cousin, 1996). À l'inverse, l'anomie s'avère particulièrement dommageable pour les élèves les plus faibles ou les plus éloignés des normes scolaires qui profitent de

2. On manque de théorie, sur le fonctionnement de l'établissement scolaire, qui inviterait à observer tel paramètre a priori pertinent quant aux progressions ou à la socialisation des élèves. La pertinence des dimensions retenues ne peut être démontrée qu'ex post, par leurs effets sur ce qu'on cherche à expliquer, ce qui donne à l'analyse une tonalité empiriste peu satisfaisante. L'absence de théorie fragilise aussi les imputations causales que l'on peut faire à partir des corrélations observées. 
l'absence de cadre normatif pour développer toutes sortes de comportements « déviants » préjudiciables à leur réussite et à toute socialisation scolaire (Cousin, 1998). Et ce d'autant plus qu'ils constituent le public majoritaire: il existe une relation entre la concentration d'élèves défavorisés en situation d'échec scolaire et l'importance des incivilités et des violences, ou au contraire des situations de repli (van Zanten, 2000b). Ce dernier constat invite à explorer davantage les relations entre «effet établissement» et composition sociale de son public, sur lesquelles nous reviendrons plus loin. Au niveau du lycée, les établissements dont les résultats se dégradent se caractérisent par de mauvaises relations entre enseignants, ce qui nuit à un partage des expériences et nourrit un certain pessimisme quant aux possibilités des élèves (Cousin, 1996).

Tous ces résultats sont bien évidemment situés. En particulier, et ce point est très important, le poids relatif des effets établissement et des effets de l'origine sociale individuelle de l'élève est dépendant de la variabilité de ces deux types de facteurs (y a-t-il plus de différences entre les établissements qu'entre les familles?). C'est ainsi que Heyneman (1986) montre que dans les pays pauvres, le contexte scolaire serait plus important que les facteurs familiaux, notamment parce que la variabilité du premier est plus forte que celle des seconds. De même, les facettes de l'établissement qui font des différences en matière d'efficacité ne seront pas forcément les mêmes en France que dans les pays anglo-saxons parce que certaines d'entre elles varient moins entre les établissements français qu'entre les établissements de ces pays.

\section{Un courant de recherche fort discuté}

Dans tous les pays, les résultats des travaux de type school effectiveness font l'objet d'un certain nombre d'interrogations et de critiques.

De manière globale, ces travaux ont été critiqués pour le positivisme étroit dont ils seraient empreints. On critique en particulier le fait que dans leur majorité les travaux relevant du courant de la school effectiveness définissent uniquement les «produits» de l'école en termes d'acquis scolaires. Or cette valorisation des acquis strictement scolaires peut varier selon les contextes culturels. Dans certains pays comme l'Australie (Wyatt, 1996), on valorise des objectifs plus larges (compétences pour devenir un adulte adapté, confiance en soi, rapport positif au savoir, etc.). Plus largement, l'optique du school effectiveness est parfois critiquée comme relevant d'une idéologie éducative, valorisant l'ordre, la hiérarchie, l'uniformité, le conformisme, idéologie inconsciente de son caractère particulier (Elliott, 1996).

Quoi qu'il en soit, s'il est certain que l'opérationalisation des «produits» de l'école est nécessairement réductrice, on ne peut contester l'importance des acquis scolaires, même s'ils sont loin de résumer la totalité des objectifs poursuivis par l'éducation. On peut également estimer que ce courant appréhende les processus qui agissent au niveau de l'école par des variables trop frustes, trop statiques, par des 
moyennes ou desconstructs (variables construites elles-mêmes à partir de plusieurs variables élémentaires) reposant sur des agrégations non théorisées en tant que telles. Derrière ces discussions, il y a évidemment la question des méthodes de recherche les plus adéquates pour appréhender ce que l'école «fait » aux enfants: l'opposition au courant de la school effectiveness prend souvent l'allure d'une opposition entre des «qualitativistes» et des «quantitativistes», que l'on peut juger inadéquate tant, précisément, l'analyse des effets écoles exige la mise en œuvre d'approches complémentaires, qui ne relèvent pas forcément des mêmes chercheurs, pour évaluer (quantitativement) l'existence même d'effets, puis pour éclairer (qualitativement) les processus sous-jacents.

Par ailleurs, l'absence d'investigation approfondie des processus fragilise évidemment les imputations causales possibles: mettre en évidence des corrélations entre des caractéristiques relativement «distales» des écoles et les progrès des élèves ne permet pas d'affirmer que les premières causent les secondes. En particulier, il est probable que les relations sont à double sens, entre les caractéristiques de la structure et les comportements individuels, notamment quand on se situe dans une dynamique temporelle.

En outre, les effets établissement apparaissent bien faibles (on l'a vu, de l'ordre de 8 à $9 \%$ de la variance des scores des élèves), sachant qu'une part de ce (faible) effet est attribuable aux classes fréquentées et non à l'établissement en tant que tel. Cela dit, si l'effet peut être jugé faible une année donnée, une suite d'effets faibles peut, avec le temps, déboucher sur des différentiels importants. On s'attend alors (et nombre de recherches anglo-saxonnes le confirment) à ce que ces « effets établissement » soient d'autant plus marqués qu'on monte dans le système. Pour prendre l'exemple de la France, lorsqu'on cumule les effets d'agrégation du collège et de la classe en fin de troisième (Grisay, 1997), on constate qu'environ $40 \%$ des variations du score de mathématiques, $32 \%$ du score de français, et $29 \%$ du score de connaissances civique sont liées à l'établissement ou à la classe fréquentée depuis la sixième. Ces chiffres traduisent à la fois le fait que les élèves ne sont pas répartis de manière aléatoire dans les établissements et dans les classes et le fait que ces deux «niveaux de contexte» produisent des effets cumulés spécifiques.

Un autre point de discussion concerne la distinction entre effet établissement et effet classe. Les résultats disponibles (et les débats scientifiques qu'ils suscitent) convainquent que la notion même d' « effet établissement», malgré sa vraisemblance sociologique, reste fragile, face à de non moins vraisemblables « effets classe». Elle suppose en effet, au niveau de l'établissement, l'existence d'un état d'esprit spécifique, d'un certain nombre de normes, de valeurs et d'attitudes partagées par tous les acteurs. Or la variance des attitudes et conceptions pédagogiques des enseignants d'un même établissement s'avère aussi grande que celle des enseignants au niveau national (c'est ce que Grisay observe pour la France, au niveau collège, 1997). De même, il existe au sein des écoles primaires françaises une très grande variété des attentes, des conceptions et des pratiques pédagogiques (Bressoux, 
1995): il y a «beaucoup plus de différences au sein des écoles qu'il y en a d'une école à l'autre». Deux constats qui ne confortent guère la notion d'effet établissement, du moins en France.

Ce que l'on peut néanmoins déduire des travaux français existants, c'est que les effets du contexte sont vraisemblablement plus forts au niveau de la classe pour ce qui est des progressions scolaires et plus marqués au niveau de l'établissement en ce qui concerne le déroulement des carrières et peut-être aussi la socialisation des élèves. On peut également faire l'hypothèse, au vu de la littérature anglosaxonne existante, que la composition scolaire (academic mix) serait une caractéristique surtout importante au niveau classe, la composition sociale (social mix) serait plus décisive au niveau de l'établissement. Mais globalement, la question de la supériorité des «effets classe » (et donc des «effets maître ») sur les «effets école» doit être considérée comme ouverte (Luyten, 2003).

On admet aujourd'hui qu'il faut penser ensemble ce qui se joue au sein de ces niveaux hiérarchisés de contexte (les anglophones parlent de nested hierarchical layers) que sont l'établissement et la classe (Dreeben et Barr, 1988; Gamoran et Mare, 1989). Le premier, le sociologue britannique Rutter (et al., 1979) a donné une contrepartie empirique précise à ce souci d'articulation entre niveaux, en subordonnant l'influence spécifique, sur les élèves, de la classe (via son organisation, les pratiques des maîtres...) à des paramètres définis au niveau de l'établissement, notamment à son climat général (développant la notion d'ethos), qui cadre et contraint ce qui va se passer au niveau hiérarchiquement «inférieur».

Aujourd'hui, les modèles multiniveaux sont l'instrument de ce type de démarche. L'idée centrale est que le niveau « organisationnel » supérieur joue sur l'efficacité du niveau inférieur en facilitant les formes d'organisation et de fonctionnement qui s'avèrent les plus porteuses d'efficacité. Ce qui est «produit» (output) par un niveau devient une ressource (input) pour le niveau inférieur. Ainsi, l'orientation générale de l'école vers les objectifs scolaires, l'atmosphère ordonnée, la cohérence entre enseignants, ou encore le leadership du chef d'établissement sont autant de facteurs qui vont rendre plus facile, au sein des classes de l'établissement, un enseignement structuré, des attentes élevées, le souci d'une évaluation continue des progrès des élèves. Ou encore, le temps que l'administration de l'école alloue pour chaque enseignant est ce qui va leur permettre de couvrir les programmes et d'instruire les élèves. On retrouve en l'occurrence les intuitions de Parsons (cité in Gamoran, 1989) selon lesquelles l'organisation gère l'allocation des ressources (niveau institutionnel et managérial) qui vont ensuite affecter les processus qui prennent place au niveau «technique» (la classe en l'occurrence).

Une autre interrogation quelque peu angoissante (si tant est que l'on vise à établir des régularités fiables) porte sur la stabilité des effets école. Si la plupart des résultats sont de nature instantanée, nombre de chercheurs invitent à la méfiance quant à la stabilité dans le temps des mesures d'efficacité ainsi dégagées. D'après la méta-analyse de Wyatt (1996), la corrélation entre des mesures d'efficacité faites 
plusieurs années de suite serait de l'ordre de 35-65 pour les écoles primaires, et de l'ordre de 70-95 pour les écoles secondaires. En France, «les performances d'un lycée sur trois peuvent être considérées comme radicalement différentes d'une année à l'autre » (Liensol et Meuret, 1987); au niveau collège, la corrélation entre indicateurs d'efficacité estimés d'une année sur l'autre serait assez faible (de l'ordre de 0,3-0,4, selon les estimations de Grisay). On explique cette instabilité par des facteurs tels que le turn-over des enseignants, ou d'autres, plus difficiles à cerner, tels que le succès de telle ou telle initiative pédagogique ou l'impact de facteurs de contexte plus généraux (et variables d'une époque à l'autre, ou d'un site à l'autre). Cette question de la stabilité est évidemment importante si l'on cherche à identifier les facteurs effectivement associés à l'efficacité, et non de pures variations aléatoires ou d'origine inconnue.

De plus, autre fragilité de la notion d'établissement, peut-on affirmer qu'il existe des « effets» qui seraient valables pour tous les élèves de l'établissement, ou seulement pour certains d'entre eux (ce qui renvoie à la notion d'efficacité différentielle), et qui par ailleurs soient constants dans toutes les disciplines? Sur ce dernier point (qu'on désigne sous le terme de consistance de l'effet établissement), il s'avère que les écoles sont souvent plus efficaces dans certaines disciplines que dans d'autres, ou encore davantage eu égard aux progressions académiques qu'en ce qui concerne lessocial outcomes (intégration sociale des élèves, civisme, développement social ou attitudes diverses). Autrement dit, seule une minorité d'écoles (entre 25 et $30 \%$ ) serait efficace (ou inefficace) dans tous les domaines.

De plus, les résultats récents (résumés in Wyatt, 1996) montrent que les écoles sont effectivement differentially effective: elles seraient plus efficaces pour certains élèves que pour d'autres; en d'autres termes, même si tous les élèves gagnent à fréquenter une école efficace, certains en tirent un profit plus important que d'autres (l'inverse valant pour les écoles inefficaces). Mais cette question reste relativement ouverte et la consistance des travaux de type differential effectiveness est médiocre; ainsi, il n'est pas véritablement établi que les écoles ont plus d'impact sur les progressions des élèves les plus faibles même si cela a été tenu pour une tendance avérée depuis les travaux de Coleman, et si ce résultat se retrouve couramment dans les travaux français (Grisay, 1997, Trancart, 1993). Par contre, certains travaux américains (résumés in Teddlie et Reynolds, 2000) montrent au contraire que les effets établissements sont plus marqués chez les élèves les plus forts (qui «profitent» tout particulièrement des écoles efficaces, où les résultats seraient de ce fait plus inégalitaires que dans les écoles inefficaces). Mais la plupart des recherches concluent que ces effets différentiels restent au demeurant modestes et que les écoles qui s'avèrent efficaces pour les uns le sont aussi pour les autres; il resterait alors justifié de produire des indicateurs globaux d'efficacité, au niveau d'une école. Toujours est-il que sous cet angle, la question de l'efficacité est très liée à celle de l'équité. Nous reviendrons dans la seconde partie sur la question (distincte) de l'efficacité différentielle des écoles selon les caractéristiques sociales agrégées de leur public. 
Le fait que la littérature sur la school effectiveness soit émaillée de résultats contradictoires reste au demeurant troublant. Il s'agit parfois de fausses contradictions, quand on rapproche des résultats établis à des niveaux scolaires différents (et portant alors souvent sur des acquis de base pour les premiers niveaux, sur des tâches de complexité supérieure aux niveaux plus élevés). Parfois, les contradictions s'expliquent par des différences méthodologiques entre travaux: les mesures d'efficacité sont très sensibles à ces variations dans les méthodes (comment les données ont été agrégées, si on a ou non tenu compte du contexte social de l'école c'est-à-dire de son school mix, de la taille des échantillons, ou encore comment on a estimé la valeur ajoutée de l'école [Wyatt, 1996]).

Il reste, c'est un autre point de discussion, qu'il est sans doute prématuré (voire contestable sur le fond) de dégager de ces travaux des lignes de conduite qu'il suffirait pour les établissements de suivre pour accroître leur efficacité; l'histoire et le contexte particulier de chaque site ne peuvent être ainsi considérés comme accessoires - l'efficacité est donc toujours contextualisée -, et des établissements distincts peuvent atteindre des niveaux comparables d'efficacité par des voies différentes. Cette question a clairement des incidences politiques, mais elle reste relativement polémique au niveau scientifique, comme l'illustrent les débats autour de l'importance de la composition du public dans l'efficacité globale d'un établissement.

\section{Effet établissement/effet du school mix}

La mouvance de la school effectiveness se centre sur l'influence, sur l'efficacité des écoles, de caractéristiques définies seulement au niveau de l'école, notamment des caractéristiques matérielles comme sa taille ou ses ressources, mais aussi des variables de nature plus psychosociale comme son climat. On entend ainsi montrer ce que l'acte d'enseignement (et son efficacité propre) doit au contexte immédiat dans lequel il s'insère, aux niveaux de la classe et de l'établissement. Il est certes légitime pour un champ de recherche de se donner des limites, de borner ses investigations. Mais il n'en est pas moins discutable de négliger le fait que l'établissement luimême est «niché » dans un environnement social plus large (un quartier, une «communauté »...). Certains travaux de ce courant font mention du «contexte» de l'établissement, désignant par-là, en particulier, le type de public accueilli. Mais, jusqu'à une date récente, il ne s'agit là que d'un cadre qui n'affecte pas la recherche de facteurs d'efficacité relativement universels et décontextualisés.

À partir des années quatre-vingt, la question apparaît clairement, tant chez certains chercheurs du courant lui-même (cf. notamment Teddlie et al., 1989) que chez certains des sociologues les plus critiques à son encontre (notamment Thrupp, 1995) de savoir s'il ne convient pas de «contextualiser» les facteurs de contexte; en d'autres termes, tous les facteurs d'efficacité dégagés jusqu'alors valent-ils quel que soit le contexte scolaire et notamment ce qu'on appelle le school mix (c'est-àdire avant tout le niveau scolaire moyen des élèves, leur profil sociologique, mais 
aussi, en arrière-plan, le caractère urbain/rural de l'environnement, la mobilisation de la communauté...)? La question est de savoir si les facteurs d'efficacité couramment dégagés ont une action propre indépendamment de la composition sociale ou scolaire du public d'élèves (avec par conséquent des possibilités d'amélioration suivant un one best way unique), ou jusqu'à quel point ils ne valent que du fait de leur association avec telle ou telle composition sociale. Dans ce dernier cas, tous les facteurs d'efficacité devraient alors être spécifiés selon le contexte; d'un point de vue politique, la portée de certaines actions ou processus pédagogiques pourrait être sur estimée, si on négligeait de tenir compte du public concerné qui en conditionne l'efficacité.

Pour preuve de la pertinence de cette interrogation, un constat troublant, souvent négligé, parfois pas même documenté de la part des chercheurs du school effectiveness, à savoir le fait que les établissements performants sont plus souvent, en moyenne, ceux qui accueillent un public de milieu aisé. On sait depuis le rapport Coleman (1966) que tous les élèves, notamment les plus faibles, «gagnent » à fréquenter une école au public plutôt favorisé, en terme de progression du moins. Coleman allait même jusqu'à dire que, de fait, les «ressources» apportées par le public d'élèves s'avéraient plus efficaces (bénéfiques pour les progressions) que les ressources de l'école elle-même. Ce constat de progressions d'autant plus fortes, pour tous les élèves, que l'école compte une proportion importante de condisciples de bon niveau académique et de milieu favorisé est assez constant dans la littérature anglo-saxonne. En soulignant cette importance de cet academic mix et de ce social mix, on pointe aussi, réciproquement, que la concentration dans une école d'élèves faibles ou de milieu défavorisé, tend à affaiblir la performance de tous les élèves.

Que ce soit chez les psychologues sociaux - qui parlent plutôt de peer effects -, les sociologues - qui parlent plutôt de contextual effects -, ou chez les économistes - qui utilisent l'expression de social externalities -, l'hypothèse selon laquelle le public d'élève est en lui-même, de par sa composition, une ressource pour l'enseignement doit donc être considérée comme confortée. Mais il faut noter que, eu égard à la distinction classique entre academic mix et social mix, la première facette du school mix est plus explorée que la seconde.

La notion même d'effet du school mix doit être précisée: il résulte de caractéristiques définies à la fois sous une forme individuelle et sous une forme agrégée (l'origine sociale des élèves par exemple), qui vont produire des effets de composition: les écoles scolarisant les élèves individuellement les plus faibles auront des performances moyennes mécaniquement amoindries, ou un groupe sera faible parce que les élèves qui le composent sont eux-mêmes faibles. Ce n'est qu'au-delà qu'on pourra éventuellement observer des effets spécifiquement contextuels. Concrètement, sur des élèves ayant, individuellement, telle origine sociale (ce qui affecte leur réussite, leurs attitudes...), est-ce que le seul fait qu'ils évoluent dans un groupe où telle ou telle origine sociale est dominante (enfants d'ouvriers scolarisés dans un établissement ouvrier par exemple) exerce, au-delà des effets de l'ori- 
gine sociale individuelle, une influence spécifique, la variable contextuelle venant redoubler ou contrarier la variable individuelle?

\section{Les effets avérés du school mix, au niveau de l'établissement}

Dans les pays de l'OCDE ( $c f$. OCDE, 2001), les performances des élèves du niveau collège sont en moyenne plus fortes dans les établissements fréquentés par des élèves de classe moyenne ou aisée. Mais des recherches spécifiques sont nécessaires pour attester l'existence d'effets contextuels, découlant de l'academic ou du social mix. Ainsi, en mathématiques et en sciences (d'après l'enquête TIMSS de 1995 exploitée par Vandenberghe, 2001), les performances des élèves sont d'autant meilleures, toutes choses égales par ailleurs, que leurs camarades de classe sont de milieu social ou de niveau scolaire élevés. Cet effet de la tonalité sociale ambiante sur les performances, même s'il s'avère souvent moins marqué que l'effet du niveau scolaire des pairs ( cf. par exemple Dar et Resh, 1986), reste néanmoins élevé, parfois presque aussi fort que l'effet individuel de l'origine sociale ( $c f$. par exemple Caldas et Bankson, 1997).

On retrouve ce type de résultat en France (cf. notamment Grisay, 1995). L'hétérogénéité du public d'élèves est également associée à plus d'efficacité et surtout à plus d'équité (moindre écart entre les «forts» et les «faibles»): les élèves des collèges à population hétérogène tendent après deux ans à se ressembler davantage; à l'inverse, les différences de performance s'accentuent dans les collèges dont la population était au départ relativement homogène. La composition du public d'élèves influence donc non seulement les performances moyennes mais aussi leur dispersion.

Il reste que dans l'ensemble, les travaux du courant de la school effectiveness ont été conduits sur des échantillons d'écoles relativement homogènes, plutôt urbaines et défavorisées; cela a certes l'intérêt de montrer clairement qu'avec un public de ce type, les écoles s'avèrent inégalement efficaces, mais cela ne favorise par une interrogation sur l'articulation avec le school mix puisqu'il est peu varié. Cette particularité des échantillons vient redoubler la relative ignorance de ces questions dans le courant de la school effectiveness, produite par son autonomisation et sa coupure avec le milieu des sociologues.

Plusieurs types de recherches sont susceptibles d'éclairer le jeu entre effets établissement et effets du school mix. On peut tout d'abord examiner si les facteurs associés à plus d'efficacité sont identiques dans les écoles les plus favorisées et les plus défavorisées. Une étude spécifique de Teddlie (et al., 1989) montre tout d'abord que certaines caractéristiques des écoles associées à l'efficacité valent pour toutes les écoles, quel que soit leur social mix; c'est le cas pour la polarisation sur des objectifs académiques, un climat d'ordre, l'importance donnée au temps actif d'apprentissage et la fréquence des évaluations des progrès des élèves. Par contre, un certain nombre de différences distinguent les écoles efficaces de classe moyenne et 
les écoles efficaces de milieu populaire. Il s'agit en particulier des facteurs suivants:

- Les attentes concernant les élèves sont élevées à la fois pour ce qui est des attentes de résultats immédiats et les attentes à plus long terme dans les écoles favorisées, alors que dans les écoles populaires, on met davantage l'accent sur les attentes immédiates.

- Les écoles efficaces de milieu favorisé ont des principaux de type manager qui laissent une certaine autonomie à leurs enseignants, alors que dans les écoles efficaces moins favorisées, les principaux sont davantage des entrepreneurs qui lancent des innovations pour changer l'école et suivent de plus près le travail des professeurs, notamment sur tout ce qui concerne la mise en ouvre des programmes dans les classes.

- Toujours pour contraster les deux situations, on éprouve moins le besoin de mettre en avant la réussite académique (son importance dans la vie par exemple) dans le premier cas, alors qu'on le fait davantage dans le second.

- Dans les écoles efficaces de milieu favorisé, on couvre un programme allant audelà des acquis de base alors qu'on ne s'y aventure que si ces acquis de base sont effectifs, dans les écoles moins favorisées.

- Dans les premières, la participation des parents est recherchée alors que dans le second, il se peut qu'au contraire on cherche à préserver l'école de toute pression (nuisible) de la communauté environnante.

Évidemment, malgré leur intérêt, ces résultats ne documentent qu'incomplètement la question des relations entre school mix et efficacité, puisqu'il n'est pas exclu que les facteurs associés à plus d'efficacité dans tous les contextes restent plus ou moins faciles à instrumenter selon que le public est plutôt favorisé ou défavorisé ${ }^{3}$. Une démarche originale et très intéressante à cet égard consiste à évaluer, au sein des effets établissement, la part qui revient à la composition du public et la part spécifique qui resterait, compte tenu du public, à des facteurs généraux d'efficacité. C'est par exemple la démarche que suivent deux chercheurs belges, Opendakker et van Damme (2001). Ils précisent qu'on peut effectivement s'attendre à ce que certains des facteurs d'efficacité les mieux établis (climat d'ordre et de discipline, bonne couverture des programmes, soutien des familles, professeurs expérimentés...) soient plus présents dans les écoles au public favorisé, et qu'en omettant de tenir compte du school mix, on surévalue l'influence potentielle de ces facteurs scolaires.

D'où leur démarche consistant à expliquer la performance en mathématiques d'élèves du secondaire flamand, en tenant compte à la fois de certains processus scolaires et du school mix (dans ses dimensions scolaires et sociales) de l'établissement. Ils construisent des variables globales de processus telles que le degré de

3. Les tenants du school improvement le reconnaissent aujourd'hui : les écoles populaires ont beaucoup plus d'efforts à faire que les écoles au public favorisé pour réunir les conditions de l'efficacité (pour une synthèse, $c f$. Teddlie et Reynolds, 2000). Dans une première phase, elles ont à mettre en place les conditions minimales de l'efficacité (environnement calme et sûr, attentes élevées, pression en vue des résultats scolaires), conditions réunies d'emblée dans les écoles favorisées; ensuite seulement, elles pourront comme les secondes s'engager sur des voies d'amélioration à plus long terme. 
coopération entre enseignants, la polarisation sur la discipline et les acquis disciplinaires, l'attention portée aux différences entre élèves, le caractère ordonné du climat. Dans un second temps, ils observent que ces processus ne sont pas également répandus selon la tonalité sociale du public: plus de coopération et de climat ordonné dans les écoles au public fort et favorisé, plus d'attention portée aux différences entre élèves dans les écoles au public faible et défavorisé, tandis que la polarisation sur les acquis scolaires est plus répandue dans les écoles au public hétérogène. Dans un troisième temps, ils examinent les relations entre ces caractéristiques «processuelles» de l'établissement et les progressions des élèves: ces dernières sont plus fortes quand le climat est ordonné, mais de manière générale, ce sont des effets d'interaction qui sont observés: la coopération entre enseignants débouche par exemple sur plus de progressions chez les élèves forts, et sur des progressions moins bonnes chez les plus faibles (sans doute par une dynamique d'effets d'étiquetage confortée par la coopération elle-même).

D'ores et déjà, on voit que les facteurs associés à plus ou moins d'efficacité ne sont pas forcément les mêmes selon les types d'élèves. Enfin, et c'est là l'apport le plus spécifique de leur travail, les chercheurs introduisent dans un modèle à la fois ces variables de processus et les variables décrivant le school mix; on observe alors que l'effet des premières est sensiblement affaibli; en particulier, l'effet positif d'un environnement ordonné diminue fortement, ce qui révèle que cette caractéristique n'apparaissait comme un facteur d'efficacité que par son association avec un public de bon niveau et favorisé. De fait, dans cette recherche centrée sur les progressions en mathématiques au niveau du secondaire, l'academic mix s'avère plus important que le social mix, sachant que ces deux variables sont très liées; nous l'avons évoqué, on retrouve ce constat d'une influence plus forte de la composition scolaire du public par rapport à sa composition sociale dans d'autres travaux (cf. par exemple Dar et Resh, 1986).

En arrière-plan, l'effet du school mix peut passer par des ressources (inégales) de l'établissement: qualité des locaux, importance du personnel, stabilité des personnes, niveau de qualification et expérience des maîtres, tous ces paramètres pouvant varier selon la composition scolaire et sociale du public; par exemple, les enseignants peuvent rechercher ou au contraire fuir tel ou tel établissement en fonction de son school mix; la stabilité (et la cohérence) des équipes est citée dans maintes recherches comme condition nécessaire à l'émergence d'un effet établissement. Peut jouer également l'appui qu'il est raisonnable d'attendre des parents. Et c'est cet ensemble plus ou moins fourni de ressources qui ferait que, selon le school mix, il est plus ou moins aisé d'organiser la vie quotidienne de l'établissement, d'y respecter les routines qui rendent le travail possible, qui permettent de ne pas perdre trop de temps pour la gestion de la discipline, les modalités mêmes de cette dernière, etc. Tout ceci peut autoriser un climat plus ou moins propice au travail et à une valorisation plus ou moins marquée des acquis scolaires des élèves, paramètres eux-mêmes vecteurs d'efficacité. 
Notons enfin que l'offre scolaire elle-même (options, dispositifs spécifiques), ainsi que les activités extrascolaires proposées aux élèves sont susceptibles de varier selon le public scolarisé. En France par exemple, on a observé que le contenu des « parcours diversifiés » proposés en collège différait sensiblement selon la composition sociale du public, avec davantage d'approches ancrées dans le «réel» pour les publics défavorisés et plus d'approches culturelles « gratuites » pour les plus favorisés (Combaz, 1999).

Au total, ces différents travaux convainquent de l'importance spécifique des variables de composition du public, sur les processus scolaires au niveau des établissements, et notamment ceux associés à des différentiels d'efficacité.

\section{Les effets du school mix au niveau de la classe ou du groupe}

De nombreux travaux anglo-saxons ont repris cette perspective au niveau de la classe et plus encore des groupes d'élèves constitués pour les activités quotidiennes d'enseignement. On recherche en particulier les effets de l'ability grouping, c'est-àdire des modes de groupement des élèves par niveau, avec constitution de groupes de niveau (le setting, en Grande-Bretagne) ou de classes homogènes d'un point de vue scolaire (le streaming en Grande-Bretagne) et du même coup, tendanciellement, social. Pour synthétiser les régularités qui se dégagent de cette littérature abondante, notons tout d'abord qu'au niveau classe, il semble que les progressions soient d'autant meilleures que le niveau scolaire initial de la classe est élevé, ce niveau moyen jouant plus que la dispersion observée à cet égard ( $c f$. en France, au niveau du collège, Pelnard-Considère, 1985, et Duru-Bellat et Mingat, 1997).

Les travaux sont moins concordants concernant l'influence de l'hétérogénéité du groupe ou de la classe. La majorité des recherches montrent néanmoins que les classes ou les groupes de niveau homogènes (et donc l'ability grouping) accroissent les inégalités scolaires entre élèves par rapport à des groupements hétérogènes. Ceci s'explique par le fait que les élèves forts regroupés dans des groupes forts progressent plus que s'ils étaient scolarisés dans un contexte hétérogène, alors qu'à l'inverse les plus faibles progressent moins ( $c f$. par exemple Kerckoff, 1986; Ireson et Hallam, 2001). Mais cet effet n'est pas symétrique et ce que «perdent» les forts dans une situation d'hétérogénéité semble moins important que ce que « gagnent» les plus faibles (de l'ordre du simple au double dans le contexte français; cf. Duru-Bellat et Mingat, 1997 ; pour l'étranger, cf. par exemple Dar et Resh, 1986; Venkatakrishan et Wiliam, 2003). D'autres travaux conduits au niveau primaire montrent que les groupes hétérogènes favorisent les élèves les plus faibles sans nuire du tout aux élèves les plus forts (Whitburn, 2001). Les effets divergents, générateurs de plus d'inégalité, d'un mode de groupement par niveau sont sans doute plus marqués dans l'enseignement secondaire.

Les élèves les plus faibles seraient donc spécialement desservis par la fréquen- 
tation d'une classe homogène faible, sachant que, de manière plus générale, ils s'avèrent plus sensibles aux effets du contexte; c'est ce que montrent certaines recherches (Duru-Bellat et Mingat, 1997, ou certains travaux de Vandenberghe), mais ce constat ne fait pas l'unanimité. Dans leur travail sur les progressions en mathématiques, Opdenakker et van Damme montrent au contraire que ce sont les élèves les plus brillants qui profitent le plus d'une scolarisation dans un contexte de niveau scolaire moyen élevé. Cette question a des incidences politiques évidentes, car si ce sont les élèves les plus faibles qui sont les plus sensibles aux caractéristiques du contexte, alors le regroupement des élèves dans des contextes hétérogènes est plus égalisant, c'est-à-dire débouche sur des performances moins inégales, que le groupement par niveau scolaire ou social. Et ce avec à la clef un niveau de performance moyen plutôt plus élevé pour l'ensemble d'une classe d'âge, puisque globalement, la résultante de ces effets contrastés et dissymétrique selon le niveau initial des élèves serait que l'hétérogénéité est associée à de meilleures progressions.

En France, dans une des premières recherches empiriques sur cette question, Mingat (1984) montre ainsi, au niveau du CP, que les progressions sont plus marquées dans les classes hétérogènes, l'effet bénéfique de l'hétérogénéité étant plus marqué pour les élèves de niveau inférieur à la moyenne (alors qu'un contexte hétérogène s'avère nuisible aux élèves très au-dessus de ce niveau moyen). Mais au total, répétons-le, la recherche internationale conclut plutôt à l'absence d'effet significatif du mode de groupement sur le niveau moyen des élèves, et à une relative inconsistance de l'effet de l'ability grouping, selon les maîtres, les disciplines (il serait plus marqué pour les mathématiques que pour la langue maternelle), et éventuellement selon l'âge des élèves (il serait plus fort chez les plus âgés). Il reste que le fait que certains «perdent» et d'autres « gagnent» à certains modes de groupement laisse prévoir des conflits d'intérêts entre les différents « usagers » de l'école.

Ces travaux restent au demeurant discutés. Aux yeux de certains économistes (cf. notamment Betts et Shkolnik, 2000), il est probable que les effets (inégalisants) de l'ability grouping ont tendance à être surestimés, dès lors que des caractéristiques non contrôlées distinguent les élèves regroupés par niveau de ceux qui fréquentent des classes hétérogènes (les économètres soulignent la fréquence de probables omitted ability bias). L'assignation des élèves aux différents groupes de niveau ne se fait en effet pas seulement à partir des résultats scolaires, mais incorpore souvent des jugements des enseignants sur la conduite, les attitudes et la motivation des élèves, ces différents paramètres étant le plus souvent moins propices à la réussite chez les élèves assignés aux groupes les plus faibles (Ireson et Hallam, 2001).

Par ailleurs, on ne saurait trop vite imputer au mode de groupement lui-même (et donc au school mix qu'il induit) les effets constatés au niveau des apprentissages, car les pratiques enseignantes et les contenus scolaires effectivement enseignés varient de fait selon le niveau des groupes. À ce propos, il est intéressant de noter que les quelques expériences de groupement par niveau qui contrôlent pré- 
cisément les contenus d'enseignement assurés (ce que permet un montage de type expérimental), ne débouchent pas sur ce type de résultat, aucune différence n’apparaissant entre les groupes, qu'ils soient homogènes ou hétérogènes (pour une synthèse, $c f$. Crahay, 2000). On observe même parfois, quand le matériel pédagogique utilisé dans les classes est strictement identique, des résultats significativement meilleurs dans les classes hétérogènes que dans les classes homogènes (Whitburn, 2001). Ceci invite évidemment à explorer les voies par lesquelles, en situation «naturelle», le school mix est susceptible d'affecter les élèves.

Avant d'aborder cette question, il faut souligner que ces résultats mettent en avant l'importance de la «variance jointe» des caractéristiques de l'école et du milieu social des élèves, en l'occurrence le fait que les élèves de milieu favorisé (et/ou de bon niveau scolaire) accèdent aux meilleures conditions d'enseignement (comme le souligne Grisay, 1997), puisque les contextes au recrutement social favorisé ou les classes de niveau moyen élevé sont les plus propices aux progressions. Cela n'est évidemment pas dépourvu d'incidences théoriques sur les sources de l'inégalité sociale à l'école: en effet, une part de l'avantage dont jouissent les enfants de milieu favorisé ne passe pas par un «héritage culturel» mais par l'accès à des contextes plus formateurs. La réciproque est vraie pour les élèves de milieu défavorisé, dont une part du «handicap » scolaire s'explique par le fait que leurs condisciples sont le plus souvent majoritairement aussi de milieu défavorisé (Caldas et Bankston, 1997).

\section{Les deux voies de l'action du school mix au niveau des groupes/classes}

Avant d'explorer les voies de l'action du school mix, un préalable est de se demander s'il agit bien en lui-même, ou s'il n'est que le reflet de processus autres ou de variables qui lui sont liées. Par exemple, une école à la tonalité sociale favorisée pourrait devoir son efficacité au fait que c'est précisément ce school mix qui y attire les enseignants les plus expérimentés et par-là les plus efficaces, ou encore que le school mix est associé à un soutien plus fort de la communauté des parents, ou encore, nous l'avons évoqué, qu'il permet de transmettre des contenus scolaires plus approfondis.

Il faudra donc s'efforcer, par des analyses multivariées, de démêler les facteurs en cause dans l'effet du school mix (et de spécifier un éventuel effet net de cette variable, résiduel, débouchant sur de nouvelles investigations). Il est vrai que toute la littérature psychopédagogique suggère nombre de mécanismes rendant compréhensible des effets spécifiques du school mix, de par toute la dynamique psychosociale qu'il induit dans les situations d'apprentissage quotidiennes. Notons que le school mix de l'établissement est vraisemblablement associé à un certain social mix de la communauté, du quartier, plus ou moins riche en «capital social» qui peut aussi peser sur l'efficacité propre de l'école; mais cette piste est largement moins explorée que 
la première. Certains travaux américains originaux (Beaulieu et al., 2001) suggèrent que la richesse en capital social de la communauté environnante, appréhendée par des indicateurs tels que le pourcentage d'adultes ayant effectivement voté ou activement engagés dans les activités religieuses, serait, toutes choses égales par ailleurs, liée à de meilleures performances scolaires chez les jeunes.

Dans les classes ou les groupes, les recherches suggèrent que les processus par lesquels opérerait l'influence du school mix sont de deux types.

\section{La quantité et la qualité de l'instruction délivrée en classe}

Les recherches anglo-saxonnes montrent à l'envi combien les attitudes et les pratiques pédagogiques des maîtres sont largement structurées par le mode de groupement des élèves auxquels ils ont affaire (Ireson et Hallam, 2001). D'une part parce qu'ils abordent les élèves avec des conceptions de ce qu'il convient de faire avec des élèves forts ou faibles. Mais aussi parce que, dans leur groupe ou leur classe, il va s'avérer plus ou moins facile, selon sa composition scolaire et sociale, de mettre en place telle ou telle pratique éducative (directive ou non par exemple), de manifester tel ou tel niveau d'exigence (dans le travail proposé, les normes du comportement en classe), de «couvrir» plus ou moins les programmes, de proposer inégalement du travail à la maison, des recherches personnelles ou des activités extrascolaires, de gérer la discipline, etc.

C'est d'autant plus probable que certains travaux ethnographiques montrent que tant les normes professionnelles des enseignants que leurs manières de faire s'élaborent dans leur contexte d'exercice quotidien. Par exemple et très concrètement, c'est le cas du type de questions qu'ils vont poser aux élèves, plus ou moins exigeantes, ouvertes, complexes, selon leurs attentes (Thrupp, 1997). La pression à réussir est plus forte envers les élèves forts, auxquels on donne aussi plus de possibilités de discussion, de réflexion et de créativité et aussi de responsabilité personnelle dans leur propre travail (Ireson et Hallam, 2001). À l'inverse, plus de passivité et de conformité sont de fait encouragées dans les groupes les plus faibles. On sait aussi que l'évaluation des performances des élèves est toujours relative au contexte de la classe (Crahay, 2000). Au total, la spécification du curriculum réel, de l'offre éducative réellement proposée, se fait donc dans un contexte concret et face à un public donné (et dans un contexte de fait décentralisé, quelle que soit la force de la centralisation formelle).

Certains travaux américains (Pallas et al., 1994) donnent un poids primordial à cette voie d'influence du school mix, qui n'est pas la plus explorée. Des méta-analyses (telle que celle de Slavin, 1990) soulignent que c'est essentiellement en ce qu'ils affectent les pratiques pédagogiques que le contexte et le mode de groupement des élèves qui le façonne (y compris d'ailleurs certaines variables «morphologiques» telles que la taille des classes) peuvent avoir un effet sur les acquisitions des élèves. 


\section{Attitudes, aspirations et comportements des élèves}

Enfin (et ce n'est sans doute pas l'effet le moins important), on peut faire l'hypothèse qu'une part de l'effet du school mix découle des interactions entre élèves (ce serait un peer effect). Les travaux des psychologues et des psychologues sociaux permettent de formuler des hypothèses: le groupe majoritaire fonctionnerait comme le groupe de référence pour tous les élèves (la comparaison sociale se ferait avec comme référence les membres de ce groupe); la composition du groupe majoritaire jouerait sur l'image de soi et les motivations des élèves à réussir, leurs représentations de l'avenir ou leurs projets, éventuellement par contraste (rappelons à cet égard que tout groupe social se définit par contraste avec les autres groupes ${ }^{4}$ ). Le groupe majoritaire jouerait également sur l'adhésion (inégale) des différents groupes aux normes scolaires, ces dernières étant plus ou moins convergentes ou plus ou moins opposées aux normes du groupe d'appartenance: réussir en classe est-il perçu comme désirable ou au contraire ridicule, par exemple, et comment le groupe renforce-t-il ou au contraire réprime-t-il tel comportement? Le contexte influe ainsi sur la réussite, en deux temps: ses caractéristiques affectent la genèse de processus psychosociaux (attitudes individuelles, normes de groupe), qui à leur tour vont influer sur les comportements et la réussite scolaires.

La composition du groupe des camarades influerait aussi sur les interactions: elles seraient inégalement stimulantes selon les différences de niveau initial entre les élèves, les élèves de milieu populaire pouvant notamment «apprendre » de leur contact avec des pairs dotés de ressources culturelles plus importantes, d'expériences de vie plus variées, ou d'expériences scolaires plus positives. Leurs visées scolaires pourraient également être stimulées par la fréquentation quotidienne d'élèves aux projets ambitieux (comme le soulignait déjà Coleman dans ses premiers travaux). Les travaux récents de Beaud (2002) illustrent sur quelques cas comment l'entrée dans un lycée de classe moyenne heurte les élèves de milieu populaire qui avaient vécu toute leur scolarité antérieure dans leur quartier, dans des écoles et collèges très ségrégués: ils souffrent d'une compétition scolaire accrue et de certaines formes d' «intimidation symbolique » de la part de leurs condisciples de milieu plus favorisé, mais dans le même temps, ils apprennent très vite des codes culturels rentables pour leurs études, avec, résultant de cette acculturation, une plus grande confiance en soi. Au total, un mode de groupement hétérogène est en général considéré comme une voie de plus grande mixité sociale.

Notons enfin qu'à côté de cette dimension cruciale de «capital social». Les aspects socioaffectifs sont également importants: les amitiés entre élèves (de niveau scolaire et social plus ou moins varié) et le soutien socioémotionnel qu'elles appor-

4. En particulier, le groupe en position défavorable, qui sent peser la menace de l'infériorisation par comparaison sociale avec le groupe le mieux place, va être contraint à une certaine créativité, pour se faire évaluer à l'aune d'autres critères qui lui seront moins défavorable (cela peut être, si un bon classement en maths ou en français s'avère hors d'atteinte, de bonnes performances en EPS, ou des déviances scolaires remarquables); sur tous ces points, cf. Vinsonneau, 1999. 
tent peuvent influer sur la performance via l'estime de soi, la confiance dans ses propres possibilités, la maîtrise du stress, les possibilités d'expression, la motivation à apprendre, etc.

Sous tous ces aspects, le contexte crée un climat normatif et psychologique qui colore de façon particulière l'expérience scolaire des élèves. Tous ces processus sont probablement, pris un à un, d'importance mineure, mais ils vont jouer de concert. L'effet du school mix est alors la résultante de nombreuses influences convergentes, de nature organisationnelle, instructionnelle ou psychosociale. On comprend alors que cet effet ne soit pas limité aux acquisitions scolaires mais se manifeste aussi sur les attitudes par rapport à l'école et aux contenus scolaires; celles-ci seraient plus favorables dans les classes hétérogènes, notamment de la part des élèves les plus culturellement éloignés de l'école. D’un point de vue psychologique, un groupement par niveau, ne serait pas sans effet en ce qu'il légitime par son existence même un traitement différencié des élèves, perçu volontiers comme une stigmatisation (Ireson et Hallam, 2001). Avec en réaction, le développement d'attitudes anti-écoles dans les groupes faibles assorties d'une moindre estime de soi. Mais ce dernier résultat est plus inconstant, certains travaux montrant au contraire que l'estime de soi des élèves les plus faibles est moins diminuée dans les groupes de niveau (faible), où la comparaison sociale est moins défavorable, que dans les groupes plus hétérogènes (Kulik et Kulik, 1992). Mais globalement, les élèves accepteraient bien un groupement par niveau, dans la mesure où cela leur semble garantir que l'enseignement va y être bien adapté à leur niveau (Ireson et Hallam, 2001).

En conclusion, on peut faire l'hypothèse (Thrupp, 1997) que derrière tous ces processus, s'expriment des jeux autour du pouvoir (inégal) des différents groupes de parvenir à contrôler le déroulement de l'instruction en classe et la qualité de cette dernière. Cela peut passer par des actions des parents d'élèves (eux-mêmes situés socialement) pour assurer à leur enfant le meilleur environnement scolaire possible (notamment l'établissement le plus efficace ou le groupe le plus profitable, quelles qu'en soient les conséquences négatives pour les autres élèves), écarter des «meilleures» classes les enseignants jugés problématiques, préserver un climat calme dans l'établissement. Dans le quotidien des classes, on sait que le curriculum réel, celui qui va s'avérer possible, est de fait négocié entre les élèves et les enseignants; ces derniers mettent en place des «stratégies de survie» (dont le groupement pas niveau, perçu comme plus facile à gérer, ferait partie). Les rapports de force autour de l'école seraient donc une des sources de l'influence du school mix. Toujours est-il que, concrètement, l'opérationalisation de la notion de « groupe majoritaire » et la description de la configuration des « groupes» en présence va s'avérer très cruciale. Au-delà du poids, important en lui-même, des élèves de milieu favorisé, les Anglo-Saxons parlent de critical mass, pour désigner le fait qu'il peut y avoir un seuil minimal pour que ce pourcentage soit assorti d'effets positifs; réciproquement, il pourrait y avoir un seuil en deçà duquel le pourcentage d'élèves 
de milieu populaire cesse d'être assorti d'effets négatifs ( $c f$. par exemple Caldas et Bankston, 1997), sachant que, à nouveau, ces questions ont des incidences évidentes et sans doute polémiques en termes de politique scolaire. Mais il faut aussi noter pour finir la dimension éthique de cette question des modes de groupement : à la fois au niveau des enseignants et à celui plus global des politiques, on défend en général des modes de groupement hétérogène pour mettre en avant le souci d'équité, alors que l'ability grouping est défendu au nom de l'efficacité et de la compétitivité; même si la recherche ne conforte guère ce dernier point de vue, il y a là des «visions du monde» cohérentes (incluant des conceptions de l'intelligence et de l'éducabilité) qui pèsent autant (voire davantage) que la recherche dans la détermination des politiques éducatives.

\section{BIBLIOGRAPHIE}

BEAUD S. (2002) 80 \% au bac... et après? Les enfants de la démocratisation scolaire. Paris: La Découverte. Beaulieu L., Israél G., Hartless G., Dyk P. (2001) For whom does the school bell toll? Multicontextual presence of social capital and student educational achievement, Journal of Socio-Economics, 30, 121-127.

BETTS J.R., SHKolnik J.L. (2000) Key difficulties in identifying the effects of ability grouping on student achievement, Economics of Education Review, 19, 21-26.

BRESSOUx P. (1994) Les recherches sur les effets-école et les effets-maitres, Revue française de pédagogie, $n^{\circ} 108,91-137$.

BRESSOUX P. (1995) Les effets du contexte scolaire sur les acquisitions des élèves: effet-école et effetsclasses en lecture, Revue française de sociologie, 36, n 2, 273-294.

Bressoux, P., Coustėre, P., Leroy-Audouin, C. (1997) Les modèles multi-niveaux dans l'analyse écologique: le cas de la recherche en éducation, Revue française de sociologie, 38, n 1, 67-96.

CALDAS S.J., BANKSton C. (1997) Effect of School Population Socioeconomic Status on Individual Academic Achievement, Journal of Educational Research, vol. 90, 5, 269-277.

CAmpbell E.Q., Alexander C.N. (1965) Structural effects and interpersonal relationships, American Journal of Sociology, 71, 284-289.

Coleman J.S. et al. (1966) Equality of Educational Opportunity. Washington D.C. : Government Printing Office.

Combaz G. (1999) Autonomie des établissements, diversification pédagogique et inégalités scolaires: effets sociaux des parcours différenciés au collège. Revue française de pédagogie, nº 128, 73-88.

Cousin O. (1993) L'effet établissement. Construction d'une problématique. Revue française de sociologie, 34, 395-419.

Cousin O. (1996) Construction et évaluation de l'effet établissement: le travail des collèges. Revue française de pédagogie, $\mathrm{n}^{\circ} 115,59-75$.

Cousin O. (1998) De l'institution à l'établissement. Le cas des collèges de banlieue. L'Orientation scolaire et professionnelle, 27, $\mathrm{n}^{\circ}$ 2, 303-325.

CRAHAY M. (2000) L'école peut-elle être juste et efficace? Bruxelles: De Boeck.

DAR Y., ReSH N. (1986) Classroom Intellectual Composition and Academic Achievement. American Educational Research Journal, 23, 3, 357-374.

Dreeben R., BARR R. (1988) Classroom composition and the design of instruction. Sociology of Education, vol.61, 129-142.

Dubet F., Cousin O., Guillemet J.-P. (1989) Mobilisation des établissements et performances scolaires. Revue française de sociologie, 30, 235-256.

DuRu-Bellat M. (2001) Controverses autour du choix de l'école: les leçons de l'étranger. L'Orientation scolaire et professionnelle, 2001, 30, $\mathrm{n}^{\circ} 2,131-153$.

Duru-Bellat M. (2002) Les inégalités sociales à l'école. Genèse et mythes. Paris: PUF. 
Duru-Bellat M., Mingat A. (1988) Le déroulement de la scolarité au collège: le contexte «fait des différences. Revue française de sociologie, 29, 649-666.

Duru-Bellat M., Mingat A. (1997) La constitution de classes de niveau par les collèges: les effets pervers d'une pratique à visée égalisatrice. Revue française de sociologie, 38, 759-790.

Elliott J. (1996) School Effectiveness Research and its Critics: alternative visions of schooling. Cambridge Journal of Education, 26, 2, 199-224.

Gamoran A., MARE R.D. (1989) Secondary school tracking and educational inequality: compensation, reinforcement, or neutrality? American Journal of Sociology, n 94, 1146-1183.

GRISAY A. (1993) Le fonctionnement des collèges et ses effets sur les élèves de $6^{e}$ et $5^{\mathrm{e}}$. Les Dossiers d'Éducation et formations, $\mathrm{n}^{\circ} 32$.

GrISAY A. (1997) L'évolution des acquis cognitifs et socio-affectifs des élèves au cours des années de collège. Les Dossiers d'Éducation et formations, nº 88.

GRISAY A. (1999) Comment évaluer la valeur ajoutée dans un système scolaire "compensatoire» ou «inégalitaire »? in D. Meuret (éd.). La justice du système éducatif. Bruxelles: De Boeck.

HEYNEMAN S. (1986) Les facteurs de la réussite scolaire dans les pays en développement, in CRAHAY M., LAFontaine D. (éds). L'art et la science de l'enseignement. Bruxelles: Labor.

JArousse J.-P., Leroy-Audoin C. (1999) Les nouveaux outils d'évaluation: quel intérêt pour l'analyse des «effets classe, in BOURdOn J., THÉLOT C. (éds). Éducation et formation: l'apport de la recherche aux politiques éducatives. Paris: CNRS éditions, 163-186.

KERCKOFF A. (1986) Effects of ability grouping in British secondary schools. American Sociological review, 51, 842-858.

Kulik J.A., Kulik C.L.L. (1992) Meta-analytic findings on grouping program. Gifted Child Quartely, $36, \mathrm{n}^{\circ} 2,73-77$.

Ireson J., Hallam S. (2001) Ability Grouping in Education. Londres: Sage.

Liensol B., Meuret D. (1987) Les performances des lycées pour la préparation au baccalauréat, Éducation et formations, $\mathrm{n}^{\circ} 11,25-36$.

Luyten H. (2003) The size of School Effects Compared to Teacher Effects: An Overview of the Research Literature. School Effectiveness and School Improvement, 14, $\mathrm{n}^{\circ}$ 1, 31-51.

Meuret D. (1995) Distribution sociale des facteurs d'efficacité des collèges, in Besse J.-M. (et al.) École efficace: de l'école primaire à l'Université. AFAE. Paris: Colin, 81-91.

Mingat A. (1984) Les acquisitions scolaires de l'élève au CP: les origines des différences. Revue française de pédagogie, nº 69, 49-63.

OCDE/OECD (2001) Knowledge and Skills for Life. First results from PISA 2000. Paris: OCDE.

OPDENAKKER M-C., VAN DAMME J. (2001) Relationship between school composition and characteristics of school process: their effect on mathematics achievement. British Educational Research Journal, vol. 27, 4, 407-431.

Pallas A., Entwisle D., Alexander K., Stluka M. (1994) Ability-Group Effects: Instructional, Social or Institutional? Sociology of Education, 67, 27-46.

Pelnard-Considère J. (1985) L'hétérogénéité des classes est-elle un handicap? L'orientation scolaire et professionnelle, 14, 1, 3-21.

Rutter M. et al. (1979) Fifteen Thousand Hours: Secondary schools and Their Effects on Children. Londres: Open Books.

SHEERENS J. (2000) Improving school effectiveness. Paris: Unesco.

SLAvin R. (1990) Achievement Effects of Ability Grouping in Secondary Schools: A Best-Evidence Synthesis. Review of Educational Research, 60, 3, 471-499.

Teddie C. Stringfield S., Wimpelberg R. Kirby P. (1989) Contextual Differences in Models for Effective Schooling in the USA, in Creemers B., Peters T., Reynolds D. (eds) School Effectiveness and School Improvement. Lisse: Swets et Zeitlinger (117-130).

Teddle C., Reynolds D. (2000) The International Handbook of School Effectiveness Research. Londres: Routledge.

Teddlie C., Stringfield S., Reynolds D. (2000) Context Issues within School Effectiveness Research, in C. Teddie et D. Reynolds (eds) The International Handbook of School Effectiveness Research. London: Falmer Press. 
ThrupP M. (1995) The «school mix» Effect: the history of an enduring problem in educational research, policy and practice. British Journal of Sociology of Education, 16, n² 2, 183-203.

Thrupp M. (1997) How School Mix Shapes School Processes: A Comparison of New Zealand Schools. New Zealand Journal of Educational Studies, 32, $\mathrm{n}^{\circ}$ 1, 53-82.

Thrupp M. (1999) Schools Making a Difference. Let's be Realistic! Buckingham: Open University Press.

Trancart D. (1993) Progrès cognitifs, non cognitifs et effet de l'établissement pour les élèves en difficulté au début du collège. Éducation et formations, n 36, 87-94.

Trancart D. (1998) L'évolution des disparités entre collèges ». Revue française de pédagogie, $n^{\circ} 124$, 43-53.

VANDENBERGHE V. (2001) Educational Equity: Social interactions may matter, in W. Hutchmacher et al. (eds), In Pursuit of Equity. Dordrecht: Kluwer Academic Publishers.

Venkatakrishnan H., Wiliam D. (2003) Tracking and Mixed-ability Grouping in Secondary School Mathematics Classroom. British Educational Research Journal, 29, n² 2, 189-204.

Vinsonneau G. (1999) Inégalités sociales et procédés identitaires. Paris: Colin.

Whitburn J. (2001) Effective classroom organisation in primart school: mathematics. Oxford Review of Education, 27, n 3, 411-428.

Wilson A.B. (1959) Residential Segregation of Social Classes and Aspirations of High School Boys. American Sociological Review, 24, 836-845

WyATт T. (1996) School effectiveness research: dead end, damp squib or smouldering fuse. Issues in Educational Research, 61 (1), 79-112.

ZANTEN (VAN) A. (2000a) Massification et régulation des établissements d'enseignement: le traitement des publics en difficulté dans les collèges de banlieue. L'Année sociologique, 50, 409-436.

ZANTEN (VAN) A. (2000b) Le quartier ou l'école? Déviance et sociabilité adolescente dans un collège de banlieue. Déviance et Société, 24, n 4, 377-401.

ZANTEN (VAN) A. (1996) Fabrication et effets de la ségrégation scolaire, in S. PAUGAM (dir.) L'exclusion, l'état des savoirs. Paris: La Découverte. 